\title{
Clinical Use of Dexamethasone Implants in Resistant Macular Edema Secondary to Branch Retinal Vascular Occlusion Compared with Intravitreal Anti-Vascular Endothelial Growth Factor
}

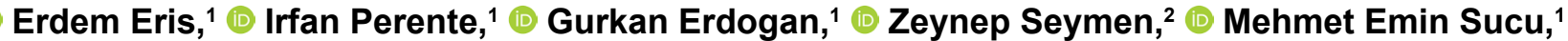

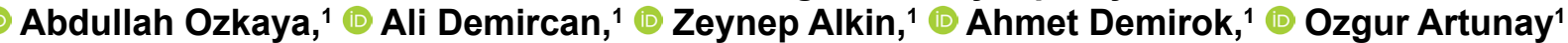

${ }^{1}$ Department of Ophthalmology, Beyoglu Eye Training and Research Hospital, Istanbul, Turkey

2Department of Ophthalmology, Suleymaniye Training and Research Hospital, Istanbul, Turkey

\begin{abstract}
Objectives: Branch retinal vein occlusion (BRVO) is the second most common type of retinal vascular disorder. Both inflammation and increased vascular endothelial growth factor (VEGF) levels play important roles in the pathogenesis of macular edema (ME) secondary to BRVO. The aim of this study was to compare the efficacy of 0.7-mg intravitreal dexamethasone implants with continued anti-VEGF treatment in patients with ME secondary to BRVO who were poor responders to at least 6 previous anti-VEGF injections.

Methods: Patients exhibiting an insufficient response to at least 6 ranibizumab treatments and who subsequently underwent a dexamethasone implant were included Group I. Patients who were at risk for cataract or glaucoma continued treatment with the same drug and were defined as Group 2. The best corrected visual acuity (BCVA) and central macular thickness (CMT) changes at month 2, 4, and 6 were measured.

Results: Ninety eyes were evaluated. In Group I, the mean baseline BCVA of $0.7 \mathrm{I} \pm 0.75$ logarithm of the minimum angle of resolution (logMAR) improved to $0.53 \pm 0.62 \log M A R$ at month $2(p<.001), 0.67 \pm 0.72 \log M A R$ at month $4(p=0.325)$, and $1.03 \pm 0.83 \log M A R$ at month $6(p=.001)$. In Group 2 , the mean baseline BCVA was $0.73 \pm 0.83 \log M A R$, and improved to $0.68 \pm 0.83 \log M A R$ at month $2(p=0.12), 0.698 \pm 0.8 \mathrm{I} \log M A R$ at month $4(p=0.270)$, and $0.76 \pm 0.80$ at month $6(p=0.546)$. The baseline CMT in each group was $588 \pm 176 \mu \mathrm{m}$ and $545 \pm 165 \mu \mathrm{m}$, respectively $(p=0.248)$. The mean CMT of Group I changed from a baseline measurement of $588 \pm 176 \mu \mathrm{m}$ to $308 \pm 132 \mu \mathrm{m}$ at month $2(p<.00 \mathrm{I}), 450 \pm 195 \mu \mathrm{m}$ at month 4 $(p<.00 \mathrm{I})$, and $510 \pm 190 \mu \mathrm{m}$ at month $6(\mathrm{p}<.00 \mathrm{I})$. The mean CMT of Group 2 changed from a baseline value of $545 \pm 165$ $\mu \mathrm{m}$ to $486 \pm 162 \mu \mathrm{m}$ at month $2(p<.001), 516 \pm 168 \mu \mathrm{m}$ at month $4(p<.001)$, and $528 \pm 171 \mu \mathrm{m}$ at month $6(p=0.037)$.

Conclusion: Dexamethasone implants were a more effective treatment for patients with BRVO-related resistant ME than ranibizumab at month 2 . However, this positive effect seems to decline rapidly in the long term.

Keywords: Branch retinal vascular occlusion dexamethasone implant, macular edema, ranibizumab.
\end{abstract}

\section{Introduction}

Branch retinal vein occlusion (BRVO) is the second most common type of retinal vascular disorder after diabetic retinopathy. Both inflammation and increased vascular endothelial growth factor (VEGF) levels play important roles in the pathogenesis of macular edema (ME) secondary to
BRVO. ME occurs as a result of blood retinal barrier (BRB) deterioration and increased vascular permeability $(\mathrm{I}-6)$. ME is the leading cause of vision loss due to $\operatorname{BRVO}(7,8)$. If ME persists, alterations formed in the macula can result in permanent loss of vision. The treatment options for eyes with vision loss due to ME associated with BRVO are focal laser photocoagulation, intravitreal steroid injection, repeated 
doses of intravitreal anti-VEGF, and vitreoretinal surgery. Bevacizumab, ranibizumab, and aflibercept are the 3 anti-VEGF agents used in the treatment of ME. Although repeated treatment with anti-VEGF therapy is primarily preferred, in some cases, an insufficient response to treatment or rebound ME can be seen. There are some studies in the literature on the subject; however, these studies did not compare continued use of the same anti-VEGF treatment $(9,10)$.

The aim of this study was to compare the efficacy of $0.7-\mathrm{mg}$ intravitreal dexamathasone implants (Ozurdex; Allergan plc, Dublin, Ireland) with continued anti-VEGF ( $0.5 \mathrm{mg}$ ranizumab) treatment in patients with ME secondary to BRVO who were poor responders to at least 6 anti-VEGF injections.

\section{Methods}

In this retrospective case control series, the medical records of patients from a single hospital who were acknowledged to have resistant ME secondary to BRVO between January 20 I 4 and June 2016 were reviewed. Patients who had received at least 6 anti-VEGF injections ( $0.5 \mathrm{mg}$ ranibizumab) within 6 months for ME associated with BRVO were included. Patients who had demonstrated inadequate anatomical (central macular thickness [CMT] $>350 \mu \mathrm{m}$ with spectral domain optical coherence tomography [OCT]) and visual (visual gain $<3$ lines) responses to at least 6 anti-VEGF treatments and who had subsequently undergone a dexamethasone implant were included in the study as Groupl. Patients with an insufficient anatomical response to at least 6 anti-VEGF treatments but had risk or predisposition for cataract, glaucoma, or central serous retinopathy and instead continued to pursue the same anti-VEGF treatment were included as Group 2 (anti-VEGF group). Insufficient visual and anatomical response to prior therapy was defined as any persisting or increasing ME observed using the Heidelberg Spectralis spectral domain OCT system (Heidelberg Engineering Inc., Heidelberg, Germany) by an expert retinal specialist (E.E).

The exclusion criteria were missed regular follow-up visits, any ocular disease other than BRVO, any ocular surgery 12 months before ME treatment, chronic disease other than hypertension, age under 18 years, cataract development during follow-up that could affect the level of visual acuity, and supplementary laser treatment within 6 months of the follow-up period.

The inclusion criteria were a minimum age of 18 years and resistant ME secondary to BRVO.

Written informed consent was obtained from all of the study patients before treatment, and the study was performed in compliance with the tenets of the Declaration of Helsinki.

\section{Study Treatment}

All of the patients were evaluated over 4 visits: A baseline assessment was performed before intravitreal therapy, and 2 , 4 , and 6 months after the initiation of intravitreal therapy. At each visit, best corrected visual acuity (BCVA) measurements using a standardized Early Treatment Diabetic Retinopathy Study chart at 4 meters, slit-lamp biomicroscopy, fundus examination, intraocular pressure measurement using Goldman applanation tonometry, and OCT imaging were conducted.

\section{Injection Procedure}

The study eye was anesthetized with topical and subconjunctival anesthetics and prepared according to standard clinical practice for intravitreal injection. In Group I, a 0.7$\mathrm{mg}$ dexamethasone implant was inserted into the vitreous cavity through the pars plana using a customized, single-use, 22-G applicator. In Group 2, $0.5 \mathrm{mg}$ ranizumab/0.5 mg/mL (Lucentis; Novartis International AG, Basel, Switzerland) was inserted into the vitreous cavity through the pars plana using a customized, single-use, 30-G needle. Patients were treated with topical $0.5 \%$ moxifloxacin $4 x$ daily starting 3 days before the day of the procedure, and for 3 days after the procedure.

\section{Outcome Measures}

The primary outcome measurement of this study was the change in BCVA. The secondary outcomes were changes in the CMT value and any complications experienced.

\section{Statistical Analysis}

Visual acuity was measured using the logarithm of the minimum angle of resolution (logMAR). The statistical evaluation was performed using repeated measures analysis of variance, paired-samples t-test, independent samples t-test, and Pearson's correlation analysis using IBM SPSS Statistics for Windows, Version 20.0. (IBM Corp., Armonk, NY, USA). P values of less than 0.05 were considered statistically significant.

\section{Results}

Ninety eyes of 90 patients were evaluated retrospectively. Group 1 and Group 2 comprised 52 and 38 patients, respectively. The mean age of the entire study population was $63.27 \pm 8.6$ years: In Group I the mean age was $62.5 \pm 9.07$ years and in Group 2 the mean age was $64.36 \pm 7.86$ years $(p=0.306)$. Forty patients were female and 50 patients were male (Table I).

Table I. Demographic data of the patients

\begin{tabular}{lcc} 
& Group I & Group 2 \\
\hline Patient count & 52 & 38 \\
Mean age (years) & $62.5 \pm 9.07$ & $64.36 \pm 7.86$ \\
Female & 23 & 17 \\
Male & 29 & 21 \\
Phakic & 38 & 27 \\
Pseudophakic & 14 & 11 \\
\hline
\end{tabular}


In Group I, the mean baseline BCVA of patients before dexamethasone implant therapy was $0.7 I \pm 0.75 \log M A R$, which improved to $0.53 \pm 0.62 \log M A R$ at month $2(p<0.00 \mathrm{I})$, $0.67 \pm 0.72 \log M A R$ at month $4(p=0.325)$, and $1.03 \pm 0.83$ logMAR at month $6(p=0.001)$. In Group 2, the mean baseline BCVA was $0.73 \pm 0.83 \log M A R$, which improved to $0.68 \pm 0.83 \log M A R$ at month $2(p=0.12), 0.698 \pm 0.81 \log M A R$ at month $4(p=0.270)$, and $0.76 \pm 0.80$ at month $6(p=0.546)$. The change in BCVA from baseline to month 6 was significantly better in the anti-VEGF group $(p=0.02 \mathrm{I})$ (Fig. I). In the dexamethasone group, the rate of aCVA gain of $\geq 3$ lines at months 2,4 , and 6 was $9.6 \%, 1.9 \%$, and $1.9 \%$, respectively. In the dexamethasone group, the rate of a stable BCVA (gain of $<3$ lines or loss of $<3$ lines) at months 2,4 , and 6 was $90.3 \%, 96.1 \%$, and $78.8 \%$, respectively. The rate of a BCVA loss of $>3$ lines in the dexamethasone group at months 2,4 , and 6 was $0 \%, 2 \%$, and $19.3 \%$, respectively. In the anti-VEGF group, the rate of a BCVA gain $\geq 3$ lines at months 2,4 , and 6 was $0 \%, 0 \%$, and $2.6 \%$, respectively. In the anti-VEGF group, the rate of a stable BCVA result (gain of $<3$ lines or loss of $<3$ lines) at months 2, 4, and 6 was $100 \%, 100 \%$, and $94.7 \%$, respectively.

The baseline CMT measurement in Group I and Group 2 was $588 \pm 176 \mu \mathrm{m}$ and $545 \pm 165 \mu \mathrm{m}$, respectively $(p=0.248)$. The mean CMT of the patients in Group I changed to $308 \pm 132 \mu \mathrm{m}$ at month $2(\mathrm{p}<0.00 \mathrm{I}), 450 \pm 195 \mu \mathrm{m}$ at month 4 $(p<0.00 \mathrm{I})$, and $5 \mathrm{I} 0 \pm 190 \mu \mathrm{m}$ at month $6(p<0.00 \mathrm{I})$. The mean CMT of the patients in Group 2 changed to $486 \pm 162 \mu \mathrm{m}$ at month $2(p<0.001), 516 \pm 168 \mu \mathrm{m}$ at month $4(p<0.001)$, and

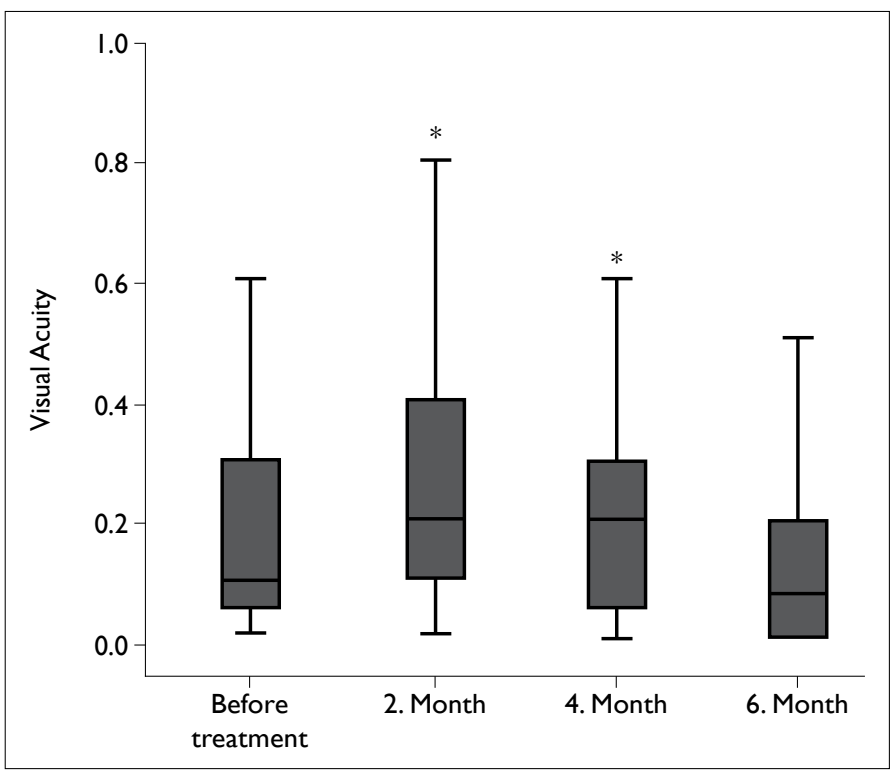

Figure I. Change in LogMAR; I) Baseline logarithm of the minimum angle of resolution (logMAR) vision; 2) LogMAR vision at the second month of treatment; 3) LogMAR vision at the fourth month of treatment 4) LogMAR vision at the sixth month of treatment; * statistically significant.
$528 \pm|7| \mu \mathrm{m}$ at month $6(\mathrm{p}=0.037)$. The change in BCVA from baseline to month 6 was significantly better in Group I $(\mathrm{p}=0.0 \mathrm{I})$ (Fig. 2).

The mean number of anti-VEGF treatments administered to patients in Group 2 in the 6-month follow-up period was $5.05 \pm 0.83$.

No serious ocular adverse events were observed. Phakic status and the presence of serous macular detachment were not correlated with the mean reduction of CMT. The mean baseline intraocular pressure (IOP) was $14.5 \pm 1.34 \mathrm{mmHg}$, the mean IOP was $15.6 \pm 2.4 \mathrm{mmHg}$ at month $2,15.5 \pm 2.05$ $\mathrm{mm} \mathrm{Hg}$ at month 4, and $15.8 \pm 2.26 \mathrm{mmHg}$ at month 6 in the dexamethasone group. Uncontrolled IOP elevation due to dexamethasone implantation was not observed in any patient. No other complications (cataract, glaucoma, etc.) were found during the 6-month follow-up.

\section{Discussion}

In our study, I dexamethasone implant led to an effective decrease in the mean CMT in cases of resistant ME associated with BRVO, but this did not correlate with the mean BCVA improvement. We also found that a single dexamethasone implant application had no significant adverse effects. It is widely thought that VEGF secretion as a result of ischemia plays a major role in the development of ME associated with BRVO. In the BRAVO (Branch Retinal Vein Occlusion) and the CRUISE (Central Retinal Vein Occlusion) studies, repeated intravitreal anti-VEGF therapy was shown to be effective in the treatment of ME associated with BRVO (II-I3). However, there are also cases of ME resistance despite repeated

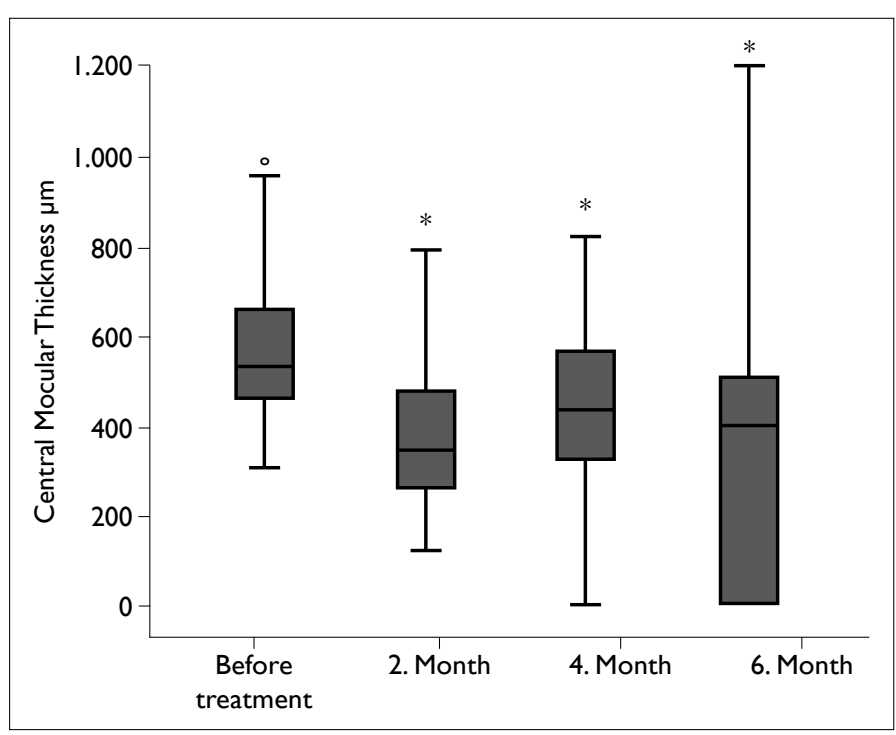

Figure 2. Change in central macular thickness; I) Baseline central macular thickness (CMT); 2) CMT at the second month of treatment; 3) CMT at the fourth month of treatment; 4) CMT at the sixth month of treatment; * statistically significant. 
intravitreal anti-VEGF therapy. In the BRAVO study, approximately $30 \%$ of patients did not achieve $20 / 40$ or better vision with repeated intravitreal anti-VEGF therapy (II). This study analyzed patients who had resistant ME secondary to BRVO. Steroids are effective in the treatment of ME associated with BRVO by inhibiting inflammation and stabilizing the BRB. Dexamethasone is a potent steroid and an intravitreal dexamethasone implant was used to treat BRVO-related ME. Thus, the cause of ME secondary to BRVO may be the role of both vascular changes and inflammation in BRVO. Therefore, to understand which pathophysiology plays a dominant role, we compared anti-VEGF and dexamethasone implant treatments for resistant ME secondary to BRVO.

Ramezani et al. (14) compared intravitreal injections of bevacizumab (IVB) and triamcinolone acetonide (IVT) and reported significant visual improvement in the IVB group compared with the IVT group. This improvement was seen 4 months after the initiation of treatment. We preferred dexamethasone implants because IVT is a short-acting drug. Pielen et al. (9) compared anti-VEGF intravitreal injections followed by dexamethasone implants, dexamethasone implant followed by anti-VEGF, and dexamethasone implants alone. However, the authors did not mention the type of anti-VEGF. The intravitreal injection interval may be different in prolonged anti-VEGF treatment, according to the type $(15,16)$. In our study, only ranizumab was used as an antiVEGF agent. Also, contrary to the study of Pielen et al., the anti-VEGF group in our study never received steroids. Chiquet et al. (I7) compared dexamethasone implants and antiVEGF (bevacizumab or ranizumab) treatment for naive ME secondary to BRVO. They reported better visual recovery in the dexamethasone group than in the anti-VEGF group at the third month of treatment. Nevertheless, no significant visual and anatomic differences were seen in the long term. In contrast to that study, we reviewed resistant ME and used only a single type of anti-VEGF. However, similarly to Chiquet et al., we saw significant visual improvement 2 months after dexamethasone implantation. Alshahrani et al. (18) reported dexamethasone implant results used in the treatment of refractory ME in retinal vascular disease of an improved visual acuity from $20 / 160$ to $20 / 80$ at I month and $20 / 60$ at 3 months $(p<0.05)$. Unlike our study, the authors compared resistant ME secondary to BRVO with resistant diabetic ME.

Some important potential complications secondary to a dexamethasone implant are cataracts, glaucoma, and endophthalmitis (19). Studies have demonstrated that the most common adverse effect in patients who underwent a dexamethasone implant was an increase in IOP $(20,21)$. Capone et al. (20) reported that although an increase in IOP of more than $10 \mathrm{mmHg}$ was seen in $32.6 \%$ of patients, only
$1.7 \%$ required incisional glaucoma surgery. The results of the GENEVA (Global evaluation of implantable dexamethasone in retinal vein occlusion with macular edema) study indicated that dexamethasone implants were effective in the treatment of macular edema secondary to BRVO, and that the percentage of eyes receiving IOP-reducing medication had increased approximately $24 \%$ in the dexamethasone implant treatment groups by day 180 (22). However, the GENEVA study did not report any superiority of dexamethasone implant treatment compared with other treatment models, such as anti-VEGF. In the present study, although we had 52 patients who underwent dexamethasone implant treatment, none of our patients needed surgery for glaucoma. In addition, we used a single type of anti-VEGF treatment as a control group; therefore, we believe our study was more specific. Wessel et al. (23) reported that during 20.3 weeks of follow-up after repeated injections of dexamethasone for the treatment of BRVO, only I (I2.5\%) patient developed significant cataracts. In their study, the patients received an average of 2.9 total injections. This indicates that a single implantation at an early stage does not lead to a significant progression to a cataract; however, repeated long-term injections significantly increases the risk of cataract progression. In our study, a statistically significant improvement in BCVA and CMT was observed in the dexamethasone group in the second month follow-up examination. Although ME had increased at the fourth-month examination it still represented an improvement from baseline values. There was no significant difference in the BCVA level measured before treatment and at month 4.

We did not reevaluate these effects because there are many studies about the long-term effects of dexamethasone. There are also many studies about dexamethasone implants and BRVO; however, our study had the largest series of patients and is the first to compare dexamethasone implant therapy with a single type of anti-VEGF therapy in resistant ME secondary to BRVO.

The primary limitation of this study is its retrospective design. Also, as this was a case control study, we could not randomize the patients for 2 drugs. We continued anti-VEGF therapy for resistant ME only when a patient was at risk for adverse effects of dexamethasone like glaucoma. Another limitation is that the anti-VEGF group underwent 3 consecutive injections but the dexamethasone group received only I injection. Strengths of our study include the large sample size and the fact that this was a single-center study. All of the patients underwent the same procedures performed by the same specialists.

To conclude, in our study, the CMT of the dexamethasone group improved to near-normal levels, and the BCVA did not reach near-normal levels at 2 months. This may be 
due to functional changes in the retina and permanent damage caused by long-lasting ME. Single-dose dexamethasone implant therapy had no clinical adverse effects. However, in long-term follow-up (>2 months), single-dose dexamethasone could not sustain the BCVA and CMT improvement as well as continued anti-VEGF therapy. After 2 months of a dexamethasone implantation, it may be beneficial to switch to anti-VEGF therapy to maintain BCVA and CMT.

\section{Disclosures}

Peer-review: Externally peer-reviewed.

Conflict of Interest: None declared.

Authorship Contributions: Involved in design and conduct of the study (EE, $A O, I P, A D)$; preparation and review of the study (GE, ZA, ZS, EE, AO); data collection (EE, MES, ZA, AD, GE, OA); and statistical analysis (EE, AD, OA, IP, ZS).

\section{References}

I. Stefánsson E. The therapeutic effects of retinal laser treatment and vitrectomy. A theory based on oxygen and vascular physiology. Acta Ophthalmol Scand 2001;79:435-40. [CrossRef]

2. Arnarsson A, Stefánsson E. Laser treatment and the mechanism of edema reduction in branch retinal vein occlusion. Invest Ophthalmol Vis Sci 2000;41:877-9.

3. Silva RM, Faria de Abreu JR, Cunha-Vaz JG. Blood-retina barrier in acute retinal branch vein occlusion. Graefes Arch Clin Exp Ophthalmol 1995;233:721-6. [CrossRef]

4. Saika S, Tanaka T, Miyamoto T, Ohnishi Y. Surgical posterior vitreous detachment combined with gas/air tamponade for treating macular edema associated with branch retinal vein occlusion: retinal tomography and visual outcome. Graefes Arch Clin Exp Ophthalmol 200I;239:729-32. [CrossRef]

5. Aiello LP, Avery RL, Arrigg PG, Keyt BA, Jampel HD, Shah ST, et al. Vascular endothelial growth factor in ocular fluid of patients with diabetic retinopathy and other retinal disorders. N Engl J Med 1994;331:1480-7. [CrossRef]

6. Noma H, Minamoto A, Funatsu H, Tsukamoto H, Nakano K, Yamashita $\mathrm{H}$, et al. Intravitreal levels of vascular endothelial growth factor and interleukin-6 are correlated with macular edema in branch retinal vein occlusion. Graefes Arch Clin Exp Ophthalmol 2006;244:309-15. [CrossRef]

7. Rogers SL, Mclntosh RL, Lim L, Mitchell P, Cheung N, Kowalski JW, et al. Natural history of branch retinal vein occlusion: an evidence-based systematic review. Ophthalmology 2010; I 17:1094-I I01.e5. [CrossRef]

8. Mclntosh RL, Rogers SL, Lim L, Cheung N, Wang JJ, Mitchell $P$, et al. Natural history of central retinal vein occlusion: an evidence-based systematic review. Ophthalmology 2010; I 17: I II3-II23.el5. [CrossRef]

9. Pielen A, Bühler AD, Heinzelmann SU, Böhringer D, Ness T, Junker B. Switch of Intravitreal Therapy for Macular Edema Secondary to Retinal Vein Occlusion from Anti-VEGF to
Dexamethasone Implant and Vice Versa. J Ophthalmol 2017;2017:583 1682. [CrossRef]

10. Lee KH, Kang EC, Koh HJ. Dexamethasone Intravitreal Implant Rescue Treatment for Bevacizumab Refractory Macular Edema Secondary to Branch Retinal Vein Occlusion. Korean J Ophthalmol 2017;31:108-II4. [CrossRef]

I I. Campochiaro PA, Heier JS, Feiner L, Gray S, Saroj N, Rundle AC, et al. Ranibizumab for macular edema following branch retinal vein occlusion: six-month primary end point results of a phase III study. Ophthalmology 20 10; I 17: I I02-I I I2.el. [CrossRef]

12. Brown DM, Campochiaro PA, Singh RP, Li Z, Gray S, Saroj N, et al. Ranibizumab for macular edema following central retinal vein occlusion: six-month primary end point results of a phase III study. Ophthalmology 2010; I 17: I 124-I I33.el. [CrossRef]

I3. Varma R, Bressler NM, Suñer I, Lee P, Dolan CM, Ward J, et al. Improved vision-related function after ranibizumab for macular edema after retinal vein occlusion: results from the BRAVO and CRUISE trials. Ophthalmology 2012;119:2108-18. [CrossRef]

14. Ramezani A, Esfandiari H, Entezari M, Moradian S, Soheilian M, Dehsarvi $B$, et al. Three intravitreal bevacizumab versus two intravitreal triamcinolone injections in recent onset central retinal vein occlusion. Acta Ophthalmol 2014;92:e530-9. [CrossRef]

15. Tagami M, Sai R, Fukuda M, Azumi A. Prolongation of injection interval after switching therapy from ranibizumab to aflibercept in Japanese patients with macular edema secondary to branch retinal vein occlusion. Clin Ophthalmol 2017; I I:403-408. [CrossRef]

16. Hanhart J, Rozenman Y. Comparison of Intravitreal Ranibizumab, Aflibercept, and Dexamethasone Implant after Bevacizumab Failure in Macular Edema Secondary to Retinal Vascular Occlusions. Ophthalmologica 2017;238: I 10-I 18. [CrossRef]

17. Chiquet C, Dupuy C, Bron AM, Aptel F, Straub M, Isaico R, et al. Intravitreal dexamethasone implant versus anti-VEGF injection for treatment-naïve patients with retinal vein occlusion and macular edema: a 12-month follow-up study. Graefes Arch Clin Exp Ophthalmol 2015;253:2095-102. [CrossRef]

18. Alshahrani ST, Dolz-Marco R, Gallego-Pinazo R, Diaz-Llopis M, Arevalo JF; KKESH International Collaborative Retina Study Group. Intravitreal Dexamethasone Implant For The Treatment Of Refractory Macular Edema In Retinal Vascular Diseases: Results Of The Kkesh International Collaborative Retina Study Group. Retina 2016;36:131-6. [CrossRef]

19. Reid GA, Sahota DS, Sarhan M. Observed Complications From Dexamethasone Intravitreal Implant For The Treatment Of Macular Edema In Retinal Vein Occlusion Over 3 Treatment Rounds. Retina 2015;35:1647-55. [CrossRef]

20. Capone A Jr, Singer MA, Dodwell DG, Dreyer RF, Oh KT, Roth DB, et al. Efficacy and safety of two or more dexamethasone intravitreal implant injections for treatment of macular edema related to retinal vein occlusion (Shasta study). Retina 20|4;34:342-5I. [CrossRef]

2I. Eter N, Mohr A, Wachtlin J, Feltgen N, Shirlaw A, Leaback R; 
German Ozurdex in RVO Real World Study Group. Dexamethasone intravitreal implant in retinal vein occlusion: real-life data from a prospective, multicenter clinical trial. Graefes Arch Clin Exp Ophthalmol 2017;255:77-87. [CrossRef]

22. Haller JA, Bandello F, Belfort R Jr, Blumenkranz MS, Gillies $M$, Heier J, et al. Dexamethasone intravitreal implant in pa- tients with macular edema related to branch or central retinal vein occlusion twelve-month study results. Ophthalmology 20I I; I I 8:2453-60. [CrossRef]

23. Wessel MM, D’Amico DJ, Kiss S. Multiple Treatments of the Sustained-Release Dexamethasone Implant in Retinal Vein Occlusion. IOVS 2012;53:2243. 